

\section{DISCLAIMER}

This report was prepared as an account of work sponsored by an agency of the United States Government. Neither the United States Government nor any agency Thereof, nor any of their employees, makes any warranty, express or implied, or assumes any legal liability or responsibility for the accuracy, completeness, or usefulness of any information, apparatus, product, or process disclosed, or represents that its use would not infringe privately owned rights. Reference herein to any specific commercial product, process, or service by trade name, trademark, manufacturer, or otherwise does not necessarily constitute or imply its endorsement, recommendation, or favoring by the United States Government or any agency thereof. The views and opinions of authors expressed herein do not necessarily state or reflect those of the United States Government or any agency thereof. 


\section{DISCLAIMER}

Portions of this document may be illegible in electronic image products. Images are produced from the best available original document. 
TABLE OF CONTENTS

$\underline{\text { Page }}$

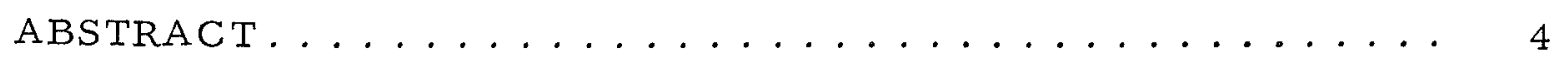

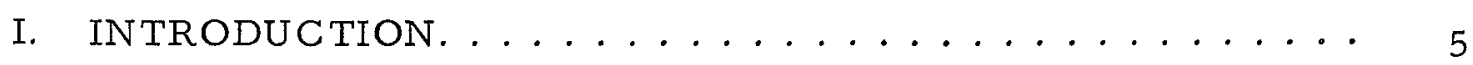

II. BOLTZMANN EQUATION AND THIN SLAB CELLS ..... 5

III. COMPARISON OF DSN, PN, AND DPN SOLUTIONS FOR

A BINARY CELL ....................... 7

IV. DISTRIBUTIONS OF DISCRETE QUADRA TURE ANGLES. . . 13

V. DISCRETE ANGLE QUADRATURES FOR THIN CELLS . . . 14

VI. SIMPLIFIED INTEGRAL TRANSPORT APPROXIMATION FOR THIN BINARY SLAB CELLS ............. 16

VII. MULTIGROUP FAST CRITICAL HETEROGENEITY

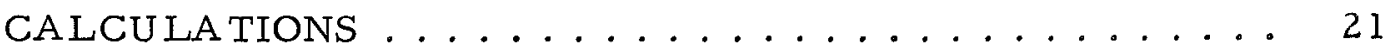

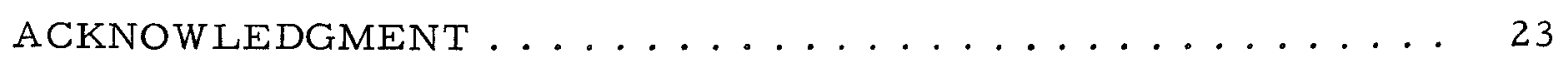

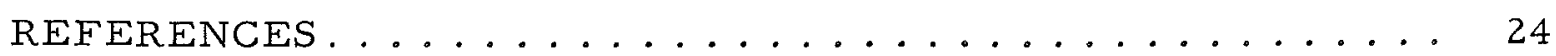




\section{LIST OF TABLES}

No.

Title

Page

I. Correspondence of Approximations with Number of

Discrete Quadrature Angles................

II. Comparison of Quadrature Constants for Case of

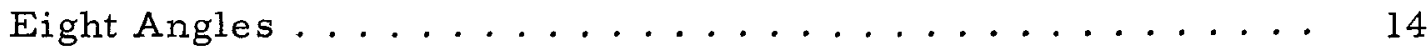

\section{LIST OF FIGURES}

No. Title

1. Half-cell Dimensions and Regional Cross Sections. . . . . . 8

2. Advantage Factors of Small Cell................ 9

3. Flux Distribution as Obtained by DP-5 Using Discrete

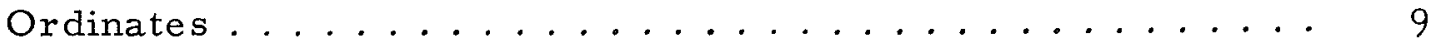

4. Vector Flux at Various Positions as Obtained by DP-5 Using Discrete Ordinates................. 10

5. Advantage Factors of Large Cell. . . . . . . . . . . 10

6. Uncollided Flux Advantage Factors of Small Cell . . . . . . 12

7. Uncollided Flux Advantage Factors of Large Cell. . . . . . 12

8. Uncollided Vector Flux at Various Positions as Obtained by DP-5 Using Discrete Ordinates............. 13

9. Uncollided Scalar Flux Distributions Showing Effects of Considering Increased Number of Neighbor Cells. . . . . . 20 


\title{
CONVERGENCE OF TRANSPORT SOLUTIONS FOR THIN SLAB CELLS
}

\author{
by
}

D. Meneghetti

\begin{abstract}
Reported(1) DSN (Discrete SN Method(2)) calculations of reactivity worths of heterogeneities in ZPR-III fast critical assemblies, due to use of various fuel plate and diluent thicknesses, have shown the necessity for high-order approximations to obtain convergence of flux shape and eigenvalue, i.e., $N=16$ solutions are generally inadequate for such cells having regional thicknesses which are fractions of mean free paths.

Convergence properties of solutions for a simplified two-region, one-energy-group, repetitive slab cell having regional thicknesses and regional cross sections representative of those encountered in some energy groups of the previous (1) three-group study are compared for $\operatorname{DSN}(N=2$, $4,8,16)$, single-spherical harmonics, $P N(N=1,3, \ldots, 11$, 13), and double spherical harmonics, $\mathrm{DPN}(\mathrm{N}=1,2,3,4,5)$, solutions for the case of a spatially constant unit source density in the alternate regions of the cell. Analogous uncollided flux solutions and an integral transport solution for the uncollided flux showing effects of the contributions of the sources in neighboring cells upon the solution arealso obtained.

As the angulax width of the anisotropic flux component occurs predominantly in the region about $\mu=0$, the "shape" of the spatial flux is largely determined by at most a few nearest-neighbor source regions, and the anisotropic component is largely the anisotropic component of the uncollided flux. Use of either a discrete ordinate method in which the quadrature angles and weights are assigned on the basis of an uncollided angular flux estimate or an integral transport method in which the angular integration is accurately carried out is suggested for more effective convergence.
\end{abstract}


For such quasi-homogeneous cells a simple handcalculational method is presented in which the spatial flux "shape" is first obtained from an uncollided flux analysis, using an integral transport treatment requiring at most a few nearest neighbor regions and arising from "effective" regional source levels, based upon the constant flux of an equivalent homogeneous cell, which include the elastic scattering sources as well as the "actual" sources. The "level" of this uncollided flux "shape" is then adjusted by a constant flux term soas to satisfy the neutron inventory requirement of total absorptions equal total "actual" sources. For multigroup solution the energy groups may be analogously treated independently by employing "effective" and "actual" regional source levels for each group based upon homogeneous cell multigroup flux levels.

\section{INTRODUCTION}

Results (1) of DSN (Discrete SN Method(2)) calculations for reactivity worths of heterogeneities in various $Z P R$-III fast assemblies have shown the necessity of having many angular directions, i.e., high $\mathrm{N}$, in order to obtain suitable convergence of flux shape and of eigenvalue. It was observed, for example, that DSN solutions with $\mathrm{N}=16$ are in general not adequate for such cells. These cells have regional thicknesses that are a fraction of a mean free path.

The present study was undertaken in order to clarify the reason for the slow convergence and to suggest possible approaches to the problem which may be more effective for these thin region cell problems.

\section{BOLTZMANN EQUATION AND THIN SLAB CELLS}

For isotropic scattering and isotropic sources the monoenergetic angular flux distribution in slab geometry is (3)

$$
\phi(X, \mu)=1 / 2 \int_{-\infty}^{X} \frac{Q\left(X^{\prime}\right)}{\mu} e^{-\frac{\left(X-X^{\prime}\right)}{\mu}} d X^{\prime} \text { for } \mu>0
$$

and 


$$
\phi(X, \mu)=1 / 2 \int_{+\infty}^{X} \frac{Q\left(X^{\prime}\right)}{\mu} e^{-\frac{\left(X-X^{\prime}\right)}{\mu}} d X^{\prime} \text { for } \mu<0,
$$

where $\mathrm{X}$ is measured in mean free path units. Further

$$
Q(X)=\frac{\Sigma_{\mathrm{S}}(\mathrm{X})}{\Sigma(\mathrm{X})} \Phi(\mathrm{X})+\mathrm{S}(\mathrm{X}),
$$

where $\Phi(X)$ is the scalar flux, $S(X)$ is the source density per mean free path, and $\Sigma_{\mathrm{S}}(\mathrm{X})$ and $\Sigma(\mathrm{X})$ are the macroscopic scattering and total cross sections, respectively.

For thin repetitive cells, such that the period of $Q(X)$ is small in comparison to the mean free path cell thickness, the exponential factors are slowly decreasing functions of $\left|\mathrm{X}-\mathrm{X}^{\prime}\right|$ over the distance of one cell thickness, except for small values of $|\mu|$. The anisotropic region of the angular flux will thus primarily occur in a small angular region about $\mu=0$.

The importance of the angles close to $\mu=0$ in characterizing the deviations of the angular flux from an isotropic variation in such thin region cells indicates that the observed slowness of the convergence with increasing degree of $\mathrm{N}$-approximation in the DSN method is due to the inefficient distribution of the discrete ordinate positions for such cells. The DSN distribution of discrete ordinates is an approximately equal spacing in $\mu$, which would require considerably high approximation in $\mathrm{N}$ to insure adequate distribution of ordinates in the small anisotropic region about $\mu=0$.

This suggests use of either a discrete ordinate method in which the discrete angles and weights of the quadrature are assigned so as to be effectively distributed, or an integral transport method in which the angular integration is accurately carried out.

As the angular flux is predominantly isotropic, except for small $|\mu|$, the spatially constant scalar flux level of an equivalent composition homogeneous cell should be an excellent flux guess from which an initial $Q(X)$ may be obtained. If the cell consists of homogeneous regions and if $S(X)$ is constant within regions, then the initial $\mathrm{Q}(\mathrm{X})$ will be a step-function distribution constant within regions. 
Integration of Eqs. (la) and (Ib) with respect to $\mu$ gives the integral transport equation for the scalar flux: (3)

$$
\phi(X)=1 / 2 \int_{-\infty}^{\infty} Q\left(X^{\prime}\right) E_{1}\left(\left|X-X^{\prime}\right|\right) d X^{\prime}
$$

where $E_{1}(y)$ is the exponential integral. Insertion of the aforementioned $Q(X)$ approximation and integration should then provide a reasonable representation of the shape of the spatial flux distribution. Furthermore it is noted, as $Q(X)$ is periodic with period short in comparison with the cell average mean free path, that, except for neighboring cell regions such that $X^{\prime}$ is close to $X$, the integral $E_{1}\left(\left|X-X^{\prime}\right|\right)$ may be considered constant for the variations of $X^{\prime}$ over any more remote cell. Thus the effective sources, $Q\left(X^{\prime}\right)$, from neighboring cell regions are largely responsible for the spatial flux shape and hence also for the anisotropic angular flux component. The effective sources in more remote cells contribute essentially only to the isotropic flux component and hence to a spatially constant flux level.

These considerations suggest a simplified integral transport approximation, amenable to hand calculation, in which the spatial flux "shape" is obtained from an uncollided flux analysis requiring at most only a few nearest-neighbor regions and arising from "effective" regional source levels, based upon the constant flux of the equivalent homogeneous cell, which include the elastic scattering sources as well as the actual sources. The "level" of the uncollided flux is then adjusted by a constant flux term to satisfy the neutron inventory of total absorptions equal total actual sources (not including elastic scattering sources).

\section{COMPARISON OF DSN, PN, AND DPN SOLUTIONS FOR A BINARY CELL}

A simplified two-region, one-energy-group, slab cell having regional thicknesses and regional cross sections of magnitudes representative of those encountered in some energy groups of the previous ( 1 ) threegroup heterogeneity study was analyzed. $\operatorname{DSN}^{(2)}(\mathrm{N}=2,4,8,16)$, Single PN $(N=1,3, \ldots, 11,13)$, and Double $P N(N=1,2,3,4,5)$ solutions were obtained for the case of a spatially constant unit source density in one region and zero source in the second region. (These are not source-iterative solutions as would be necessary for a heterogeneity calculation in an actual case; however, the main convergence features and comparisons of the methods are valid.)

The uncollided flux solutions werealso obtained for comparison with the multiply-collided solutions. In addition, the integral transport solution 
for the uncollided flux showing the effects of successive neighboring cell sources upon the overall solution was also calculated.

The single spherical harmonics (PN) solutions were obtained by a Fortran program (4) using as input the tabulated $(5,6)$ values of the deter-

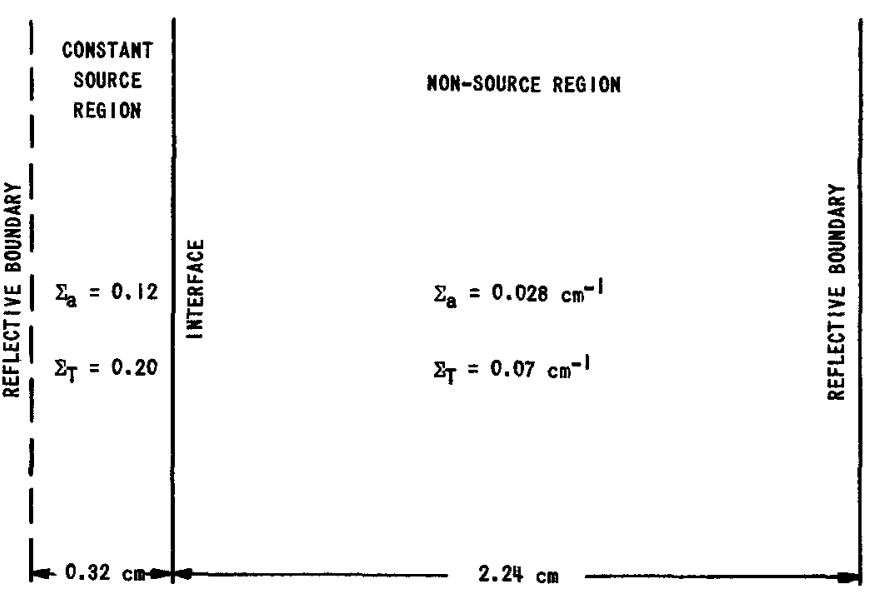

FIG. I HALF-CELL DIMENSIONS AND REGIONAL CROSS SECTIONS minantal roots $\nu_{i}$ and the functions $G_{j}\left(\nu_{i}\right)$. The double spherical harmonics (D PN) solutions were obtained by means of the Flip(7) program and the RANCH( 8$)$ program. The uncollided integral transport solution was obtained by a Fortran program. (4)

The dimensions of the half-cell and the macroscopic regional cross sections are shown in Fig. 1. In the source region the full regional thicknesses in absorption and total mean free paths were 0.0768 and 0.128 , respectively. In

the nonsource region the regional thicknesses in absorption and total mean free paths were 0.125 and 0.314 , respectively.

The ratios of the average values of the flux in the source region to the average value of the flux in the nonsource region, $\bar{\phi}_{\mathrm{S}} / \bar{\phi}_{\mathrm{ns}}$, are shown in Fig. 2 for successive approximations by the various methods of solution. Only the DP-5 solution appeared to be reasonably convergent. The spatial distribution of the scalar flux by DP-5 is shown in Fig. 3.

The vector fluxes at various positions in the cell as obtained by the DP-5 solution, which employs the method of discrete ordinates with double Gaussian quadrature with twelve discrete angles, are shown in Fig. 4. The vector flux distributions are largely isotropic except for angles close to the tangential plane of the slabs.

To obtain a set of solutions which are more converged by all these methods, the regional thicknesses of the cell were increased to ten times the dimensions shown in Fig. 1. Identical macroscopic cross sections were retained. The values of the advantage factor, $\bar{\phi}_{\mathrm{S}} / \bar{\phi}_{\mathrm{ns}}$, obtained by means of these calculations are shown in Fig. 5. The DS-16, P-13, and DP-2 gave essentially converged identical advantage factors. In this case the convergence by the DPN method was from above. 


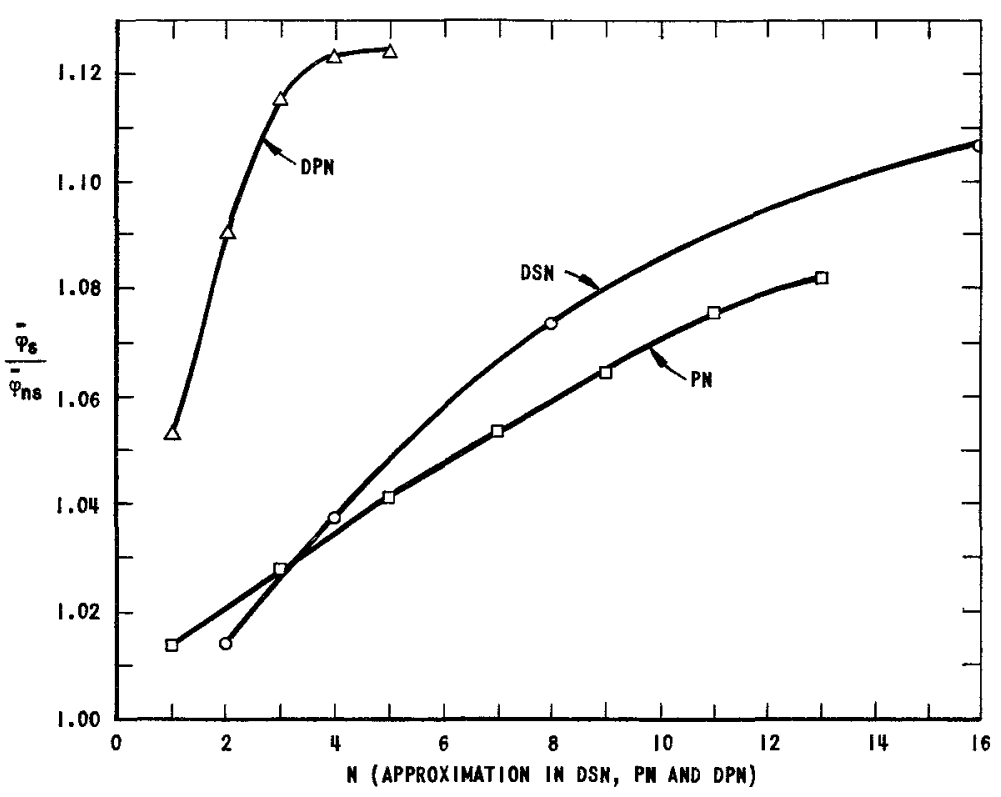

FIG. 2

ADVANTAGE FACTORS OF SMALL CELL

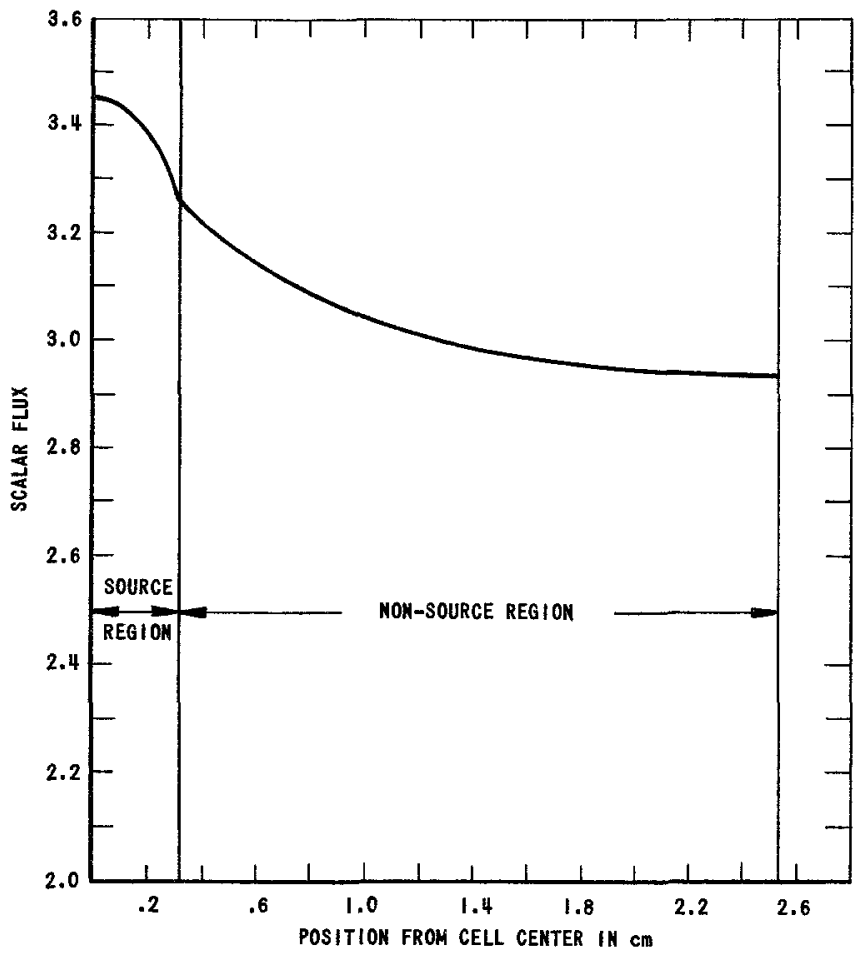

FIG, 3

FLUX DISTRIBUTION AS OBTAINED BY DP-5 USING DISCRETE ORDINATES 


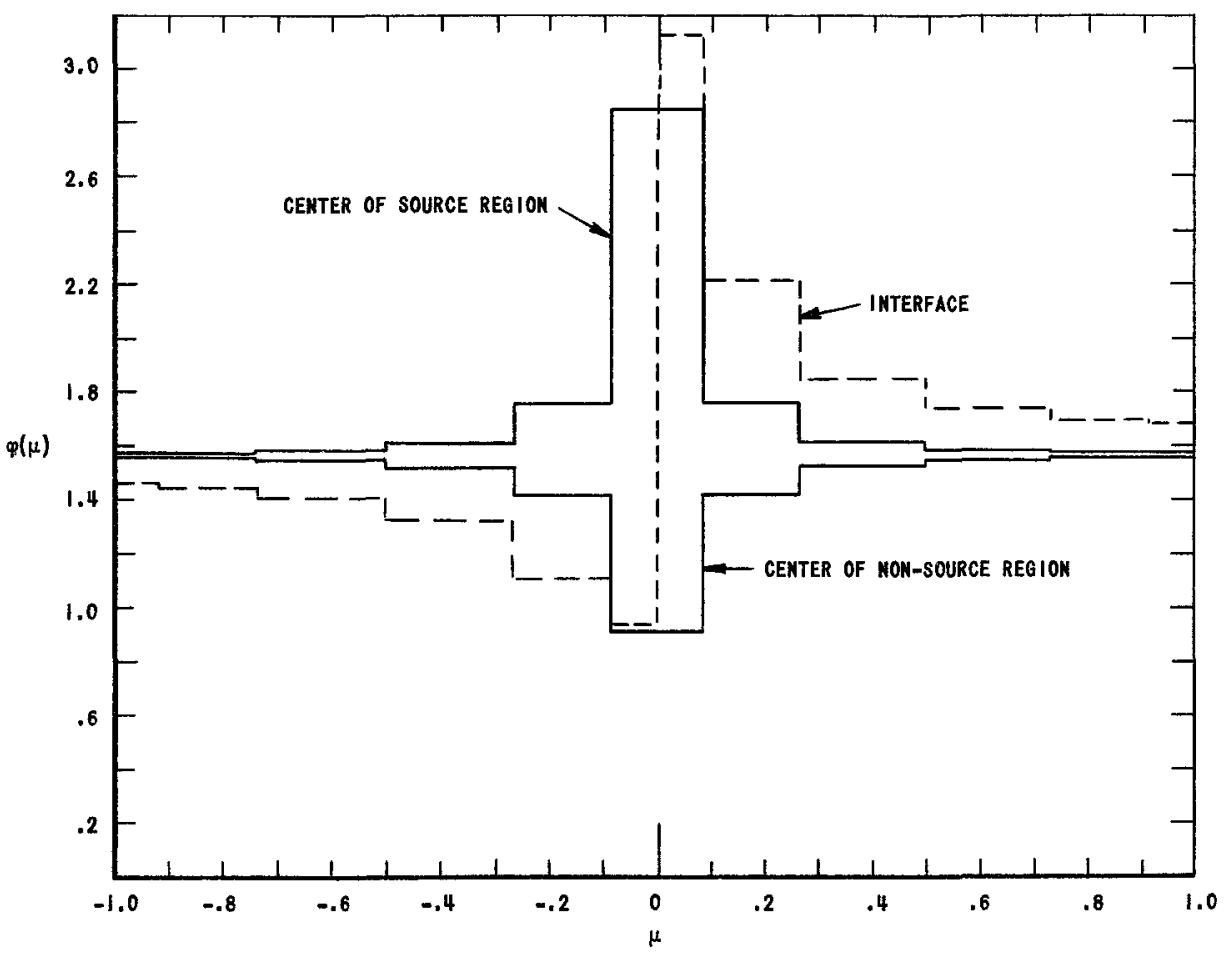

FIG. 4

VECTOR FLUX AT VARIOUS POSITIONS AS OBTAINED BY DP-5 USING DISCRETE ORDINATES

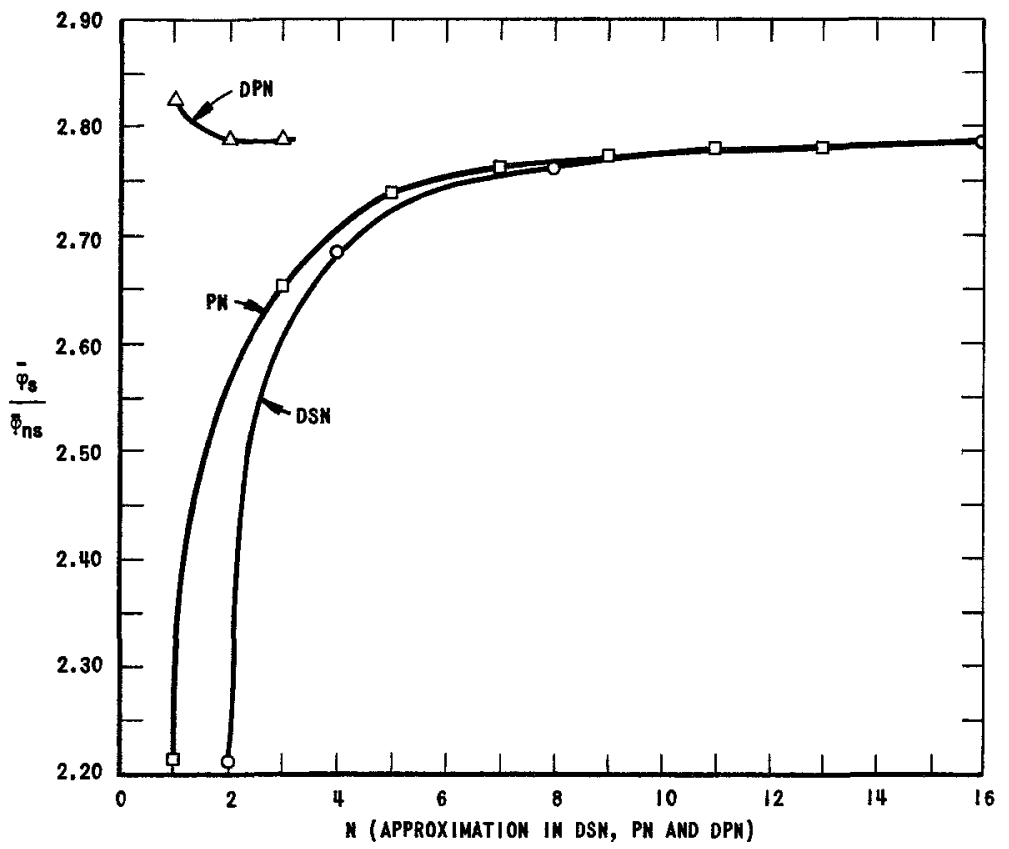

FIG. 5

ADVANTAGE FACTORS OF LARGE CELL 
Analogous calculations of the advantage factors for the uncollided flux by the various methods and, in addition, by an integral transport solution in which the effects of successively more remote source regions upon the advantage factor are observable, are shown in Figs. 6 and 7, respectively, for the two cell sizes. The uncollided fluxes are the solutions obtained by the various methods when the regional absorption cross sections are increased so as to be equal to the regional total cross sections. For the integral transport solutions the n-approximation refers to the number of neighboring source regions considered in the calculations in the following sense: The $\mathrm{n}=0$ refers to the uncollided flux in a source region due to neutrons arising from the sources in that region; and it refers to the uncollided flux in a nonsource region due to neutrons originating in the adjacent source regions on both sides of the nonsource region. Similarly, the $\mathrm{n}=1$ solution refers to uncollided flux in a source region due to neutrons from sources arising in that region and in the closest neighboring source regions on both sides; and it refers to the uncollided flux in a nonsource region due to neutrons originating in the first and second nearest source regions on both sides of the nonsource regions.

The uncollided flux advantage factors by DSN, PN, and DPN as functions of solution approximations exhibited analogous convergence shapes to the preceding multiple collision results, except that the curves were displaced to lower values of the advantage factor. The results of the uncollided flux integral transport solutions showed in this case that consideration of sources from about four to five neighboring cells on both sides must be considered in order to give reasonably convergent uncollided advantage factors. This is because sufficient neighboring cells must be considered to account for the fate of the emitted source neutrons, especially those emitted in closely perpendicular directions (to the planes) which contribute largely to the overall flux level. Insofar as the flux shape is concerned, the integral method requires in this case at most one neighboring cell to be considered.

The uncollided vector fluxes at various positions by the DP-5 solution are shown in Fig. 8. Comparison with corresponding results (see Fig. 4) for the multiple collision case showed that the angular spreads about $\mu=0$, in which the anisotropic component occurs, were approximately the same in the two cases. The two sets of angular distributions differed essentially only by the increased magnitude of the isotropic flux level in the multiple collision case. This is because in this example the neutron sources in the two regions, due to scattering, are small relative to the applied source. Inclusion of the scattering source levels, based upon the homogeneous cell constant flux level, into the uncollided flux solution will result in more improved comparison with the anisotropic component of the multiply collided solution. 


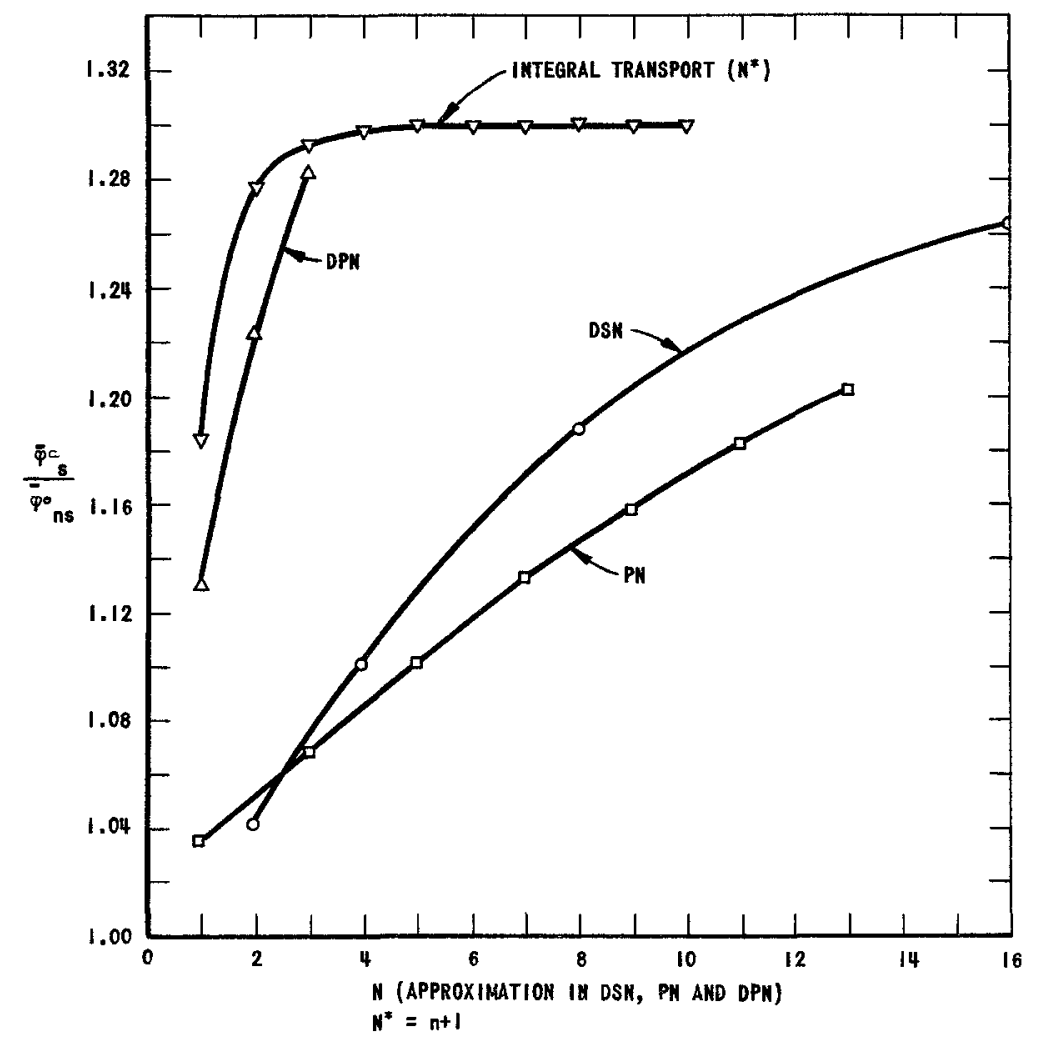

FIE. 6

UnCOLL IDED FLUX ADVANTAGE FaCtors OF SMALL CELL

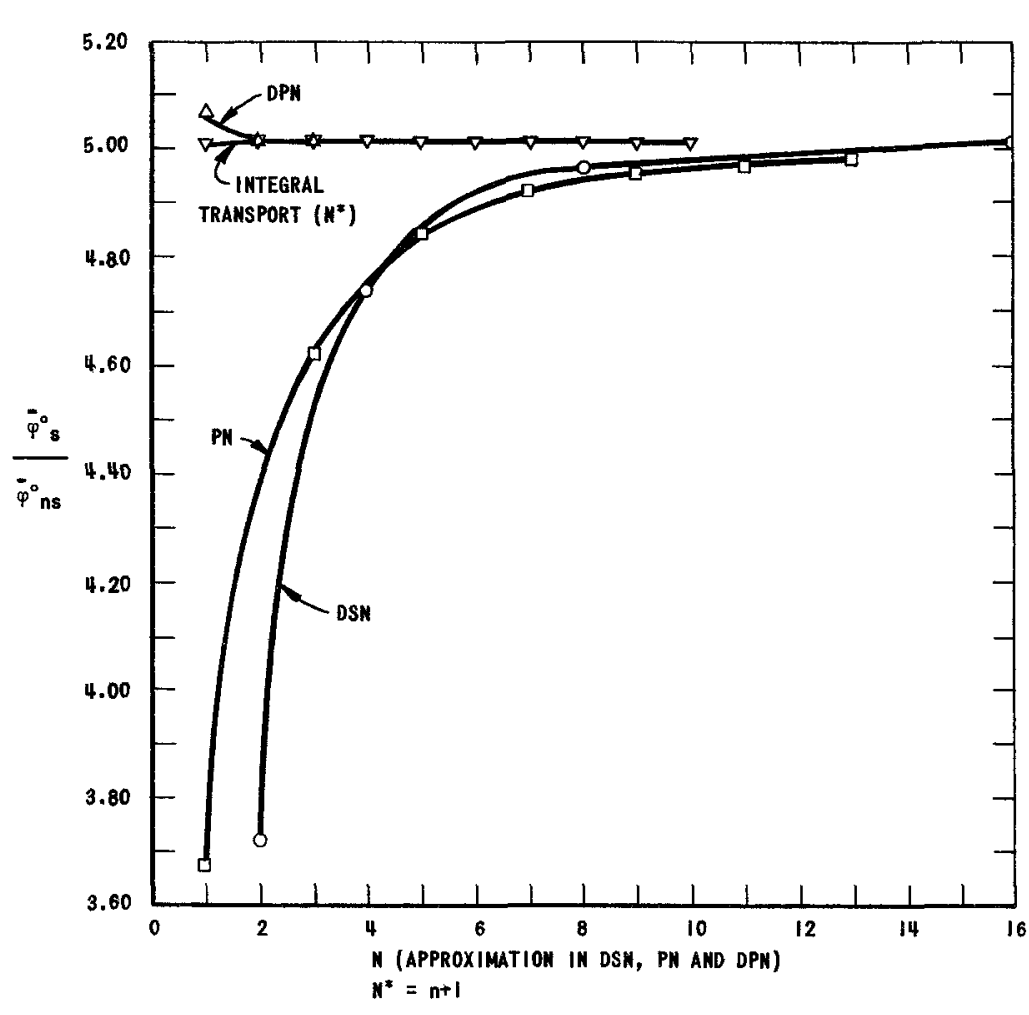

FIG. 7

UnCOLLIDED FLUX adVantaGe Factors OF LARGE CELL 


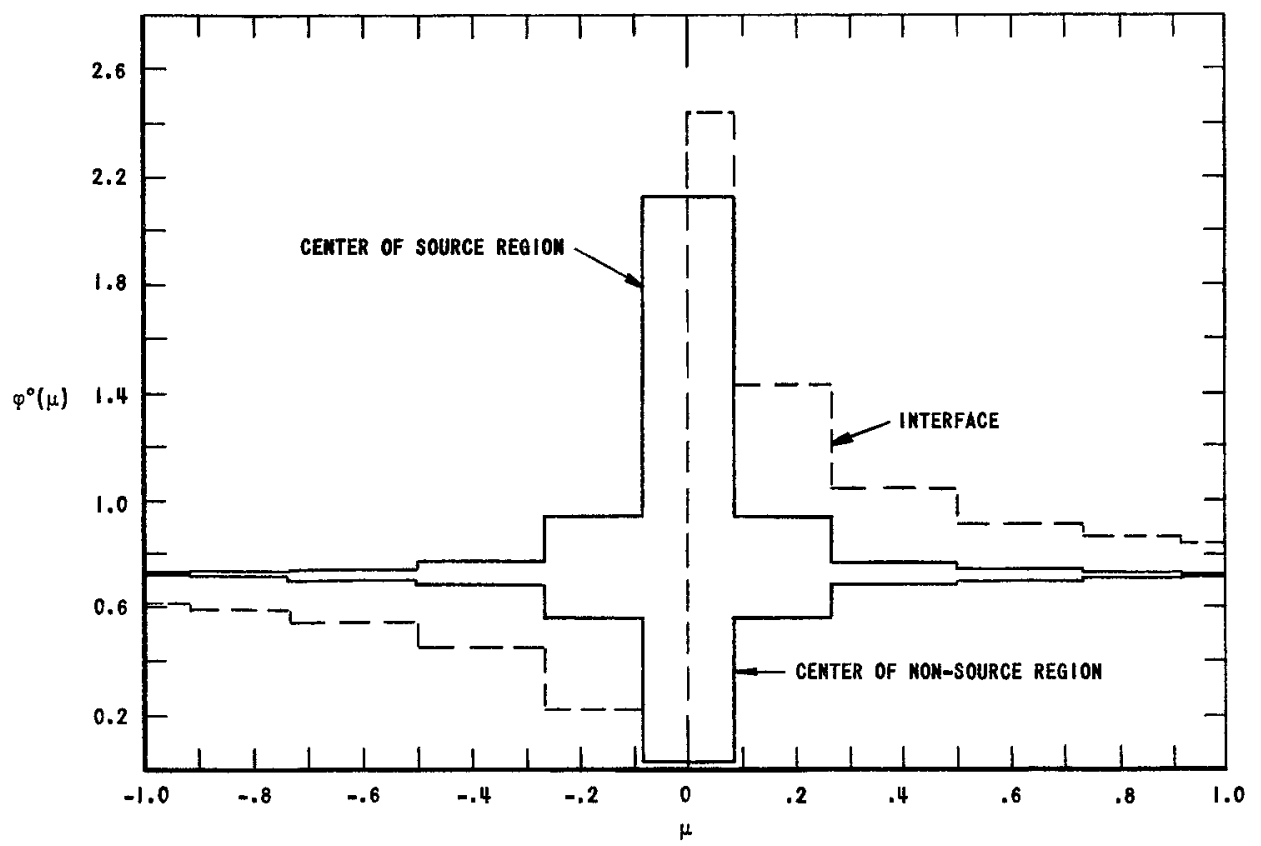

FIG. B

UNCOLLIDED VECTOR FLUX AT VARIOUS POSITIONS AS OBTAINED BY DP-5 USING DISCRETE ORDINATES

IV. DISTRIBUTIONS OF DISCRETE QUADRA TURE ANGLES

Comparison of the relative convergence rates of the advantage factors by the various methods of solution are best made by comparing the advantage factors for equivalent number of discrete angles. The correspondence follows from the equivalence of single and double Gaussian discrete quadratures with corresponding single and double spherical harmonics solutions by series expansions. $(9-11)$ The equivalence of the approximations in terms of number of discrete angles is given in Table $I$.

Table I

CORRESPONDENCE OF APPROXIMATIONS WITH NUMBER OF DISCRETE

QUADRATURE ANGLES

\begin{tabular}{|c|r|r|r|}
\hline \multirow{2}{*}{$\begin{array}{c}\text { Number of } \\
\text { Angles }\end{array}$} & \multicolumn{3}{|c|}{$\mathrm{N}$} \\
\cline { 2 - 4 } & DSN & PN & DPN \\
\hline 4 & 4 & 3 & 1 \\
6 & 6 & 5 & 2 \\
8 & 8 & 7 & 3 \\
10 & 10 & 9 & 4 \\
12 & 12 & 11 & 5 \\
\hline
\end{tabular}


For intercomparison the approximate values for the discrete angles $\left|\mu_{\lambda}\right|$ and the quadrature weighting factors $R_{\lambda}$ for the case of eight discrete angles are listed in Table II for DSN, PN, and DPN quadratures.

Table II

COMPARISON OF QUADRA TURE CONSTANTS FOR

CASE OF EIGHT ANGLES

\begin{tabular}{|l|c|c|c|c|c|c|c|c|}
\hline \multirow{2}{*}{ Quadrature } & \multicolumn{4}{|c|}{$\left|\mu_{\lambda}\right|$} & \multicolumn{4}{c|}{$\left|\mathrm{R}_{\lambda}\right|$} \\
\cline { 2 - 8 } & $\lambda=1$ & 2 & 3 & 4 & $\lambda=1$ & 2 & 3 & 4 \\
\hline $\begin{array}{l}\text { Single(11) } \\
\text { Gaussian } \\
\text { P-7 } \\
\begin{array}{l}\text { Double(11) } \\
\text { Gaussian } \\
\text { DP-3 }\end{array}\end{array}$ & 0.960 & 0.797 & 0.526 & 0.183 & 0.101 & 0.222 & 0.314 & 0.363 \\
DS-8(12) & 0.882 & 0.630 & 0.378 & 0.126 & 0.250 & 0.250 & 0.250 & 0.250 \\
\hline
\end{tabular}

The greater effectivenesses of the DSN relative to the PN and of the DPN relative to the DSN are due to the increasingly more effective distributions of the smallex discrete directions in the region near $|\mu|=0$. For the case of the cell having regional thicknesses ten times as large, the convergence rates by $P N$ and DSN were quite similar, indicating that the differences of the quadratures are not important enough, i.e., the angular spread about $|\mu|=0$ which contributes to the anisotropic flux component is relatively large. The DPN results in this case converge from above, which indicates that the distributions of the discrete directions as demanded by double Gaussian quadrature are slightly too heavily distributed toward the $|\mu|=0$ region.

\section{DISCRETE ANGLE QUADRA TURES FOR THIN CELLS}

Choice of a quadrature for use in a discrete ordinate method of solution in the case of thin cells may be decided by the angular flux distribution of an a priori uncollided flux solution including the scattering sources. As the angular width of the anisotropic component of the angular flux varies somewhat at different positions within a cell, an approximate estimate of the angular region of the anisotropic flux will probably suffice. This means roughly that for cosine of angles $|\mu| \sim\left|\mu_{\mathrm{C}}\right|=\mathrm{t} /\left(2 \lambda_{\mathrm{T}}\right)$, where $\lambda_{\mathrm{T}}$ is the total mean free path and $t / 2$ is the half thickness of the predominant source region, that the angular flux will largely deviate from an isotropic one. By 
predominant source region is meant the region having the largest source density, including the scattering sources, per regional mean free path.

This criterion is based on the realization that the flux anisotropy for a thin slab source is large along rays with small $|\mu|$ and small along rays with larger $|\mu|$ values. In the latter case the ray has a short path in the source region before entering adjacent regions. The expression for $\left|\mu_{c}\right|$ is based on the approximate assumption that at the center of the predominant source region the angular region of predominant anisotropy occurs at those angles for which the long rays are greater than one mean free path.

Care should be taken to insure that among the number of discrete directions $\left|\mu_{\lambda}\right|$ allotted for a given approximation $\left|\mu_{\lambda}\right|>\left|\mu_{c}\right|$ and $\left|\mu_{\lambda}\right| \cong\left|\mu_{c}\right|$ be included. In this regard a double Gaussian quadrature does distribute a reasonable portion of the discrete angles in the angular region of the anisotropic flux; however, it also tends to emphasize the forward and backward directions by alloting more discrete directions than relatively necessary in the isotropic flux interval where $|\mu| \gg\rangle\left|\mu_{c}\right|$.

In the example problem,

$$
\left|\mu_{\mathrm{c}}\right|=\frac{0.32 \mathrm{~cm}}{5.0 \mathrm{~cm}}=0.064 .
$$

Reasonable values of $\left|\mu_{\lambda}\right|$ and $R_{\lambda}$ for a four discrete angle quadrature might be

$$
\begin{aligned}
& \left|\mu_{1}\right|=0.064 / 2=0.032, \\
& \left|\mu_{2}\right|=0.468, \\
& R_{1}=0.064, \text { and } \\
& R_{2}=0.936 .
\end{aligned}
$$

This gives an advantage factor of 1.082, which may be compared with the corresponding four-angle DP- 1 value of 1.053 and the six-angle DP-2 value of 1.092. Certainly four angles are still not sufficient for this cell; however, it is clear that by suitable quadrature assignment, considerable improvement also at higher approximations may be obtained. 


\section{SIMPLIFIED INTEGRAL TRANSPORT APPROXIMATION FOR THIN BINARY SLAB CELLS}

An approximate solution is described in which the spatial flux "shape" is obtained from an uncollided flux solution requiring consideration of sources from at most only a few nearest-neighbor regions. The uncollided flux is considered to arise from "effective" flat regional source levels, which include the elastic scattering sources as well as applied and fission sources, based upon the constant flux level of the equivalent homogeneous cell. The "level" of the uncollided flux shape obtained from consideration of but a few neighboring source regions is then adjusted by a constant fluxterm to satisfy the neutron inventory requirement that total absorptions equal total actual sources (not including elastic scattering sources) to obtain an approximation to the multiply-collided flux distribution. If necessary for more accuracy, a source iteration may be carried out using new effective flat regional sources based upon regional average flux values obtained.

The uncollided distribution per mean free path in a region $A$ at position $\mathrm{X}_{\mathrm{A}}$ and for positive $\mu$ values due to spatially constant isotropic sources, $S_{A}$ and $S_{B}$, per unit mean free paths in two repeating regions $A$ and $B$ of a binary slab array having thicknesses $A$ and $B$ respectively in mean free path units is given by the expression

$$
\begin{aligned}
& \phi_{A+}^{0}\left(\mu, \mathrm{X}_{\mathrm{A}}\right)=\frac{\mathrm{S}_{\mathrm{A}}}{2}\left[1-\mathrm{e}^{-\left(\frac{\mathrm{X}_{\mathrm{A}}-\mathrm{A}}{\mu}\right)}+\frac{\mathrm{e}^{\frac{-\mathrm{X}_{\mathrm{A}}}{\mu}\left(\mathrm{e}^{\frac{+\mathrm{A}}{\mu}}-1\right)}}{1-\mathrm{e}^{-\frac{\mathrm{A}+\mathrm{B}}{\mu}}}\right] \\
& +\frac{S_{B}}{2}\left[\frac{e^{\frac{-X_{A}}{\mu}}\left(1-e^{\frac{-B}{\mu}}\right)}{1-\frac{\mathrm{A}+\mathrm{B}}{\mu}}\right]
\end{aligned}
$$

where $X_{A}$ is measured from the left boundary of region $A$. Exchange of $B$ for $A, A$ for $B, X_{B}$ for $X_{A}, X_{A}$ for $X_{B}, S_{B}$ for $S_{A}$, and $S_{A}$ for $S_{B}$ gives $\phi^{0}{ }_{\mathrm{B}}+\left(\mu, \mathrm{X}_{\mathrm{B}}\right)$, where $\mathrm{X}_{\mathrm{B}}$ is measured from the left boundary of region $\mathrm{B}$. Corresponding relations for negative $\mu$ are obtainable by symmetry considerations. The uncollided fluxes at positions $\mathrm{x}_{\mathrm{A}}$ and $\mathrm{x}_{\mathrm{B}}$, expressed in centimeters, are

$$
\phi_{\mathrm{A}+}^{0}\left(\mu, \mathrm{x}_{\mathrm{A}}\right) \equiv \phi_{\mathrm{A}+}^{0}\left(\mu, \mathrm{x}_{\mathrm{A}}\right), \text { where } \mathrm{x}_{\mathrm{A}}=\Sigma_{\mathrm{A}} \mathrm{x}_{\mathrm{A}}
$$


and

$$
\phi_{\mathrm{B}+}^{0}\left(\mu, \mathrm{x}_{\mathrm{B}}\right) \equiv \phi_{\mathrm{B}+}^{0}\left(\mu, \mathrm{x}_{\mathrm{B}}\right) \text {, where } \mathrm{x}_{\mathrm{B}}=\Sigma_{\mathrm{B}} \mathrm{x}_{\mathrm{B}}
$$

If $S_{A}=S_{B}$, the expressions reduce to isotropic fluxes constant throughout. Thus if $S_{A}>S_{B}$, then $\Delta S=S_{A}-S_{B}$ may replace $S_{A}$, and $S_{B}$ may be replaced by zero without altering the "shape" of the uncollided angular flux distribution except for an additional constant isotropic term throughout, which may readily be determined by the neutron inventory requirement that total first collisions be equal to total sources.

For the source $\Delta S$ in region $A$ only, the uncollided angular flux "shapes" in regions $A$ and $B$ are given by the expressions

$$
\phi_{A+}^{0}\left(\mu, X_{A}\right)=\frac{\Delta S}{2}\left[\frac{\left(1-e^{-X_{A} / \mu}\right)\left(e^{\left.-\frac{A+B}{\mu}-e^{-\frac{X_{A}+B}{\mu}}\right)}\right.}{1-e^{-\frac{A+B}{\mu}}}\right]
$$

and

$$
\phi_{\mathrm{B}+}^{0}\left(\mu, \mathrm{X}_{\mathrm{B}}\right)=\frac{\Delta \mathrm{S}}{2}\left[\frac{\mathrm{e}^{-\mathrm{X}_{\mathrm{B}} / \mu}\left(1-\mathrm{e}^{-\mathrm{A} / \mu}\right)}{1-\mathrm{e}^{-\frac{\mathrm{A}+\mathrm{B}}{\mu}}}\right] \text {, }
$$

respectively.

If the regions are fractions of mean free paths thick, $A / \mu$ and $\mathrm{B} / \mu$ are sufficiently large only for $\mu$ values close to zero, so that the relations then reduce to

$$
\begin{aligned}
& \phi_{\mathrm{A}+}^{0}\left(\mu, \mathrm{X}_{\mathrm{A}}\right) \cong \frac{\Delta \mathrm{S}}{2}\left[1-\mathrm{e}^{-\mathrm{X}_{\mathrm{A}} / \mu}\right] \\
& \phi_{\mathrm{B}+}^{0}\left(\mu, \mathrm{X}_{\mathrm{B}}\right) \cong \frac{\Delta \mathrm{S}}{2}\left[\mathrm{e}^{-\mathrm{X}_{\mathrm{B}} / \mu}\left(1-\mathrm{e}^{-\mathrm{A} / \mu}\right)\right]
\end{aligned}
$$

for these small $\mu$ values. For $\mu$ values such that $(A+B) / \mu$ is small, Equations 2 and 3 reduce to isotropic flux distributions.

If $A$ and $B$ are thin but $B<A$, then more nearest-neighbor cells are important in determining the nonisotropic angular flux spread in the 
angular region about $\mu=0$. In terms of the scalar flux distribution the "shape" requires more nearest-neighbor cells to be considered (as distinct from flux-level adjustment alone). Suitable quadrature designations may be estimated from the preceding relationships for use in a discrete ordinate method of solution.

Consideration of both positive and negative-directed source neutrons together with integrations over $\mu$-space, rather than proceeding by taking limits of geometric series, lead to the following expression for the uncollided scalar fluxes in the two regions:

$$
\begin{aligned}
\phi_{A}^{0}\left(X_{A}\right)= & \frac{\Delta S}{2}\left\{2-E_{2}\left(X_{A}\right)-E_{2}\left(A-X_{A}\right)\right. \\
& +\sum_{n=1}^{\infty}\left[E_{2}\left(X_{A}-A+n A+n B\right)-E_{2}\left(X_{A}+n A+n B\right)\right. \\
& \left.\left.+E_{2}\left(-X_{A}+n A+n B\right)-E_{2}\left(A-X_{A}+n A+n B\right)\right]\right\}
\end{aligned}
$$

and,

$$
\begin{aligned}
\phi_{B}^{0}\left(X_{B}\right)= & \frac{\Delta S}{2}\left\{E_{2}\left(X_{B}\right)-E_{2}\left(A-X_{B}\right)+E_{2}\left(B-X_{B}\right)-E_{2}\left(A+B-X_{B}\right)\right. \\
& +\sum_{n=1}^{\infty}\left[E_{2}\left(X_{B}+n B+n A\right)-E_{2}\left(A+X_{B}+n B+n A\right)\right. \\
& \left.\left.+E_{2}\left(B-X_{B}+n B+n A\right)-E_{2}\left(A+B-X_{B}+n B+n A\right)\right]\right\}
\end{aligned}
$$

where

$$
E_{n}(\alpha)=\int_{0}^{1} \mu^{n-2} e^{-\alpha / \mu} d \mu
$$

Because of spatial symmetry, only $A / 2 \leq \mathrm{X}_{\mathrm{A}} \leq \mathrm{A}$ and $0 \leq \mathrm{X}_{\mathrm{B}} \leq \mathrm{B} / 2$, corresponding to half cells, need be evaluated.

The mean values of the uncollided fluxes are

$$
\begin{aligned}
\bar{\phi}_{A}^{0}= & \frac{\Delta S}{A}\left\{A-0.5+E_{3}(A)\right. \\
& \left.+\sum_{n=1}^{\infty}\left[E_{3}(-A+n A+n B)-2 E_{3}(n A+n B)+E_{3}(A+n A+n B)\right]\right\}
\end{aligned}
$$


and

$$
\begin{aligned}
\bar{\phi}_{B}^{0}= & \frac{\Delta S}{B}\left\{0.5-E_{3}(B)-E_{3}(A)+E_{3}(A+B)\right. \\
& \left.+\sum_{n=1}^{\infty}\left[E_{3}(n A+n B)-E_{3}(B+n A+n B)-E_{3}(A+n A+n B)+E_{3}(A+B+n A+n B)\right]\right\} .
\end{aligned}
$$

Neglect of the terms in the summations gives the uncollided flux in the source region $A$ due to neutrons arising from sources in that region and the uncollided flux in the nonsource region $B$ due to neutrons originating in the first adjacent source regions on both sides of the nonsource region, etc., as previously described. It should be noted that, because of this described pairing of the flux solutions in source and nonsource regions, that an apparent flux discontinuity, $\phi_{\mathrm{B}}^{0}(0)-\phi_{\mathrm{A}}^{0}(\mathrm{~A})$, will occur at the region interface for non"level"-converged solutions. This discontinuity becomes increasingly negligible with consideration of sufficient terms so as to satisfy the neutron inventory requirement of total sources equal to total first collisions. Insofar as the flux "shapes" in the separate regions are concerned, the shape convergence as distinct from the overall level convergence will generally converge rapidly, so that in many cases even neglect of all but $n=0$ terms may be suitable. Correction for the flux discontinuity at the interface may then be carried out by subtraction of $\phi_{\mathrm{B}}^{0}(0)-\phi_{\mathrm{A}}^{0}(\mathrm{~A})$, evaluated for the given approximation, from the flux or mean flux of region B. Adjustment of the few-neighbor uncollided flux to the many-neighbor multiply-collided flux is then made by addition of a constant flux term determined from the inventory requirement that total absorptions equal total actual sources.

The number of neighboring source regions necessary to obtain a suitable uncollided flux "shape" may be determined by noting when

$$
\Delta \bar{\phi}_{\mathrm{n}}^{0} \equiv \bar{\phi}_{\mathrm{n}_{\mathrm{A}}}^{0}-\bar{\phi}_{\mathrm{n}_{\mathrm{B}}}^{0}-\left[\phi_{\mathrm{n}_{\mathrm{B}}}^{0}(0)-\phi_{\mathrm{n}_{\mathrm{A}}}^{0}(\mathrm{~A})\right]
$$

remains essentially constant with increasing integer $n=0,1, \ldots$. The quantity in the bracket corrects for the interface discontinuity effect previously referred to.

Adequacy of the uncollided scalar flux shape to approximate the multiply- collided flux shape may be tested by comparing the relative magnitudes of the average first-collision source differences, $\left|\bar{S}_{A}^{1}-\bar{S}_{B}^{1}\right|$, with the initially assumed $\Delta S$, where

$$
\bar{S}_{R}{ }_{R}=\bar{\phi}_{R}^{0}\left[\frac{{ }^{\Sigma} s_{R}}{{ } T_{R}}\right]
$$


for region $R$. Here $\bar{\phi}_{R}^{0}$ is the mean uncollided flux in region $R$ after correction for the flux discontinuity effect and adjustment in level by an overall constant uncollided flux term determined by the first collision inventory requirement that total first collisions equal total actual sources.

For the example cell the uncollided flux shapes by the various n-approximations for the case of unit intensity per centimeter applied source in the thinner region are shown in Fig. 9.

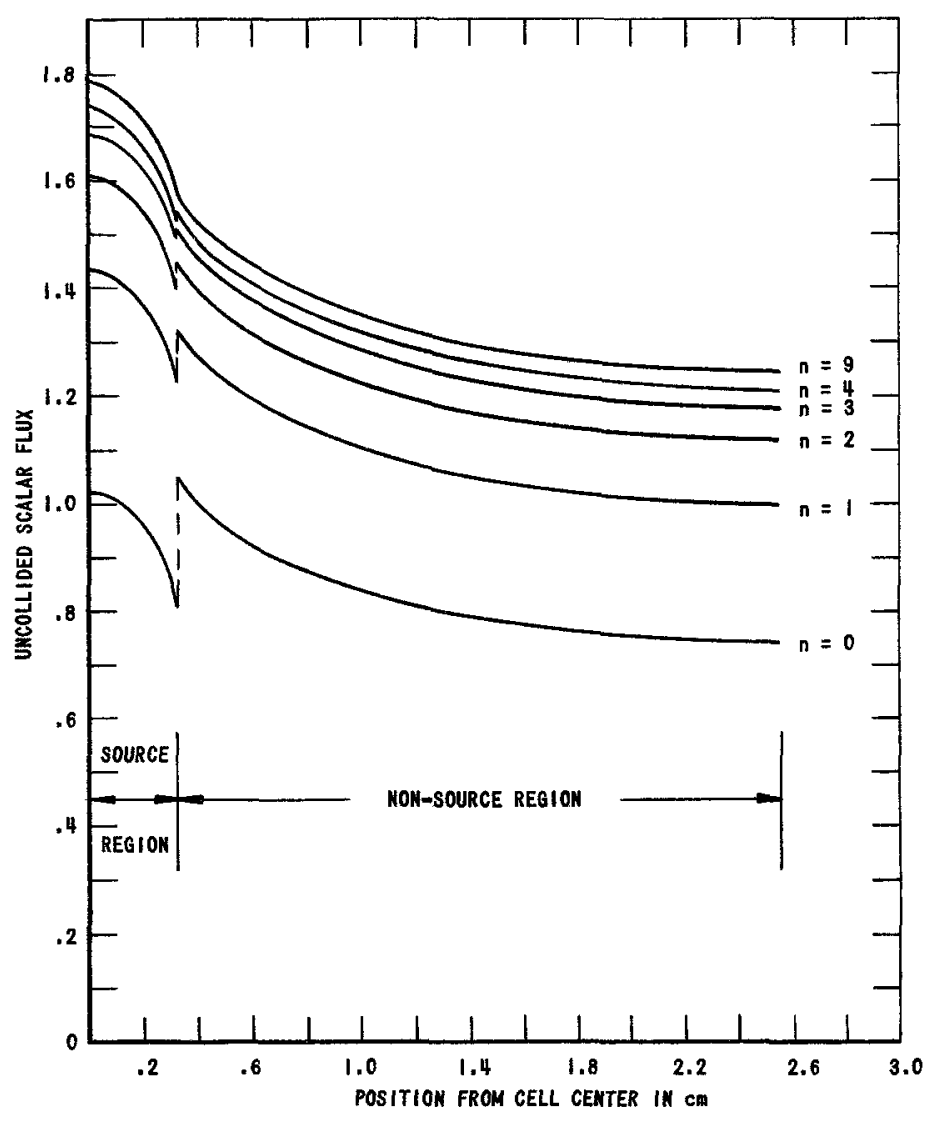

FIG. 9

UNCOLLIDED SCALAR FLUX DISTRIBUTIONS SHOWING EFFECTS OF CONSIDERING INCREASED NUMBER OF NEIGHBOR CELLS

The elastic scattering sources have not been included here. It is seen in this case that the $n=0$ approximation gives an excellent flux shape in both regions, so that by enforcement of continuity at the interface and addition of a suitable overall constant flux term the many-neighbor uncollided flux is well approximated. In addition, because the effect of the elastic scattering sources upon flux shape is small in this case, a further addition of an overall constant flux term results in good agreement with the multiplecollision DP-5 distribution shown in Fig. 3. In general, it is necessary, however, to determine the flux shape by an uncollided analys is based upon the effective sources. 
Calculation of the fractional change in multiplication constant, relative to a homogeneous one, due to plate configuration heterogeneities in quasihomogeneous systems may be carried out analogously. It is suggested that for a multigroup solution the energy groups be treated independently. This neglects the effects of the spatial shapes of the group fluxes insofar as the coupling between groups is concerned; and it assumes that the spatially integrated group fission and group slowing-down sources over the cell are identical to the group sources of the equivalent homogeneous cell. Initially as sumed constant sources should be the "effective" sources including also the sources due to scattering within a given group. In the previous example the cross sections of the two regions were such that the contribution to the "effective" regional sources from $\Sigma_{s_{r}} \phi$ homogenizedcell could be neglected. The "effective" source per total mean free path for Group $\mathrm{j}$, Region $\mathrm{r}$, is to be taken as proportional to

$$
S_{r, j}^{e f f}=\frac{\sum_{k=1}^{J} \beta_{j}\left(\nu \Sigma_{f}\right)_{r, k} \phi_{k}^{\text {homo }}+\sum_{k=1}^{j-1} \sum_{k \rightarrow j} \phi_{k}^{\text {homo }}+\sum_{j \rightarrow j} \phi_{\text {total }}, j}{\sum_{r, j}^{\text {homo }}},
$$

where $\phi_{k}^{\text {homo }}$ is the $k^{\prime}$ th group normal mode solution for the equivalent homogeneous cell. (Details of accounting for the overall neutron leakage and for the deviations from infinite slab geometry are described in Reference 1.)

In general, by the uncollided flux shape scheme of solution used is meant the uncollided flux "shape" based upon the "effective" source levels as defined above. For "level" adjustment of the uncollided flux "shape", however, actual sources must be employed, as defined by

$$
\mathrm{s}_{\mathrm{r}, \mathrm{j}}^{\text {actual }}=\sum_{\mathrm{k}=1}^{\mathrm{J}} \beta_{\mathrm{j}}\left(\nu \Sigma_{\mathrm{f}}\right)_{\mathrm{r}, \mathrm{k}} \phi_{\mathrm{k}}^{\text {homo }}+\sum_{\mathrm{k}=1}^{\mathrm{j}-1} \Sigma_{k \rightarrow j} \phi_{k}^{\text {homo }}
$$

where $s_{r, j}^{\text {actual }}$ is the actual source per centimeter. It is noted that the apparent sources due to group in-scattering are not included.

Application to a three-group analysis for $\left(\Delta \mathrm{k}_{\mathrm{eff}} / \mathrm{k}_{\mathrm{eff}}^{\text {homo }}\right)$ of a binary slab array consisting of $0.16-\mathrm{cm}$ thick enriched fuel plates separated by about $3.53 \mathrm{~cm}$ of diluent may be compared with the analogous results obtained by a DS- 16 calculation. (1) The atomic density (units of $10^{24} / \mathrm{cm}^{3}$ ) 
composition of a fuel plate region is assumed: $\mathrm{N}^{\mathrm{U}^{235}}=0.0394$ and $\mathrm{N}^{\mathrm{U}^{238}}=0.00296$. Atomic density composition of a diluent region is assumed: $\mathrm{N}^{\mathrm{U}^{235}}=0.0000128, \mathrm{~N}^{238}=0.006407, \mathrm{~N}^{\mathrm{Fe}}=0.02144$, and $\mathrm{N}^{\mathrm{Al}}=0.02162$. A core having this composition represents approximately a large $U^{235}$-fueled fast assembly having a spherical critical mass of about $1,000 \mathrm{~kg}$, assuming a reflector saving of $20 \mathrm{~cm}$. To allow direct comparisons of group flux advantage factors with corresponding DS-16 results, (1) the identical regional cross sections and energy groups are retained. The groups have lower limits: $1.35 \mathrm{Mev}, 9.12 \mathrm{kev}$, and $1.0 \mathrm{kev}$.

tors, $\bar{\phi}_{\mathrm{j}_{\text {fuel }}}$ The separately calculated (nonsource iterative) group advantage facing DP-5 factors were 1.17 and 0.92 for Groups 1 and 3, respectively. Corresponding DS-16 factors (sources and three groups iterative) were 1.13, 1.00, and 0.93.(1) The Group 3 factors do not affect the heterogeneity estimate, because the Group 3 flux is negligible for this composition. For Group 1 the predominant source region is the thin fuel plate region. The advantage factors were larger by the simplified integral transport approach and by DP- 5 than by DS-16, in effect, because the latter has its minimum discrete ordinate at $|\mu|=0.0626$, which is somewhat large to be encompassed within the region of predominant angular flux anisotropy for such a thin source region

The value of [ $k$ hetero $k_{\text {homo }}$ / khomo obtained was about +0.006 , which may be compared with the DS-16 value (1) of +0.0043 . For a system of equivalent composition having $0.32-\mathrm{cm}$ thick fuel plates separated by $7.06 \mathrm{~cm}$ of diluent the values were +0.011 and $+0.0100(1)$ respectively.

In the case of Group 3, the predominant "source" region, because of neutron transfer from Group 2 to Group 3, is the diluent region whose group thickness is about one total mean free path, and the intervening absorption region (fuel plate) is a fraction of a mean free path. To obtain reasonably suitable uncollided flux "shape" convergence requires an $\mathrm{n}=1$ nearest-neighbor approximation in contrast to an $n=0$ for the first two groups; however, in addition, the "shape" of the uncollided flux spatial distribution is not closely representative of the multiple-collided "shape," so that the value 0.94 is larger than the DP-5 converged value of 0.92 .

If heterogeneity cases are encountered for which the overall multiplication constant is too sensitive to the ratio of the average regional group fluxes for energy groups having such large "effective" sources, subsequent collision sources must be considered. A division of the large "source" region into smaller subdivisions to insure that the assumption of constant regional "effective" sources do not affect the group advantage factor, to the extent of affecting the cell eigenvalue, may be necessary. In these instances it would seem more advantageous to use the variational 
method (13) for these groups in conjunction with appropriately chosen trial functions for the diluent source region.

Thus the simplified integral transport approximation is most accurate and useful for thin cells having very thin predominant effective source regions. These cells are characteristic of fast reactor critical assemblies fueled by thin fuel plates; although the method may be useful for calculation of disadvantage factors in cases of thermal reactor cells having very thin water gaps.

\section{ACKNOWLEDGMENT}

The author wishes to thank Dr. H. H. Hummel for his interest and for numerous valuable comments on various aspects of the study. Thanks are also due to Dr. E. H. Bareiss for helpful discussions on discrete ordinate methods. The author is indebted to Mr. G. J. Duffy for permission to use his exponential integral evaluation program in advance of publication. 


\section{REFERENCES}

1. D. Meneghetti and M. F. Loomis, Calculation of Heterogeneity Effects in ZPR-III Fast Assemblies Using the DSN Program, ANL-6218(1960).

2. B. Carlson et al., The DSN and TDC Neutron Transport Codes, LAMS-2346 (1960).

3. A. W. Weinberg and L. C. Noderer, Theory of Neutron Chain Reactions, AECD-3471 (1951).

4. D. Meneghetti (unpublished).

5. J. C. Mark, The Spherical Harmonics Method, II, CRT-338, (May 1945 and June 1947).

6. A. Jeffrey, Tables of Characteristic Roots and Functions for the Spherical Harmonics Method, Math. Tables \& Better Aids to Comp., XII, 321 (Oct. 1959).

7. B. L. Anderson et al., Flip and BM-704 Code to Solve the PL and Double PL Equations in Slab Geometry, WAPD-TM-134 (March 1959).

8. L. A. Hageman and J. T. Mandel, RANCH - AnIBM-704 Program Used to Solve the One-dimensional Single-energy Neutron Transport Equation with Anisotropic Scattering, WAPD-TM-268.

9. R. D. Richtmyer, Difference Methods for Initial-Value Problems, Interscience Publishers, Inc., New York (1957).

10. E. H. Bareiss, A Survey and Classification of Transport Theory Calculation Techniques, Proc. of the Second United Nations Conference on the Peaceful Uses of Atomic Energy, Geneva (1958), Vol. 16, P/503.

11. E. H. Bareiss, Flexible Transport Theory Routines for Nuclear Reactor Design, Report 1030, David Taylor Model Basin (Dec. 1956).

12. B. Carlson, Numerical Solution of Transient and Steady-state Neutron Transport Problems, LA-2260 (Oct. 1959).

13. L. S. Bohl et al., Variational Calculations of Lattice Self-Shielding, Nuc. Sci. \& Eng. 4, p. 257 (1958) 